\title{
Terminal Sliding Mode Control with Unidirectional Auxiliary Surfaces for Hypersonic Vehicles Based on Adaptive Disturbance Observer
}

\author{
Naibao He, ${ }^{1,2}$ Changsheng Jiang, ${ }^{1}$ Bin Jiang, ${ }^{1}$ and Qian Gao ${ }^{3}$ \\ ${ }^{1}$ College of Automation Engineering, Nanjing University of Aeronautics and Astronautics, Nanjing 210016, China \\ ${ }^{2}$ School of Electrical and Information Engineering, Jiangsu University of Technology, Changzhou 213001, China \\ ${ }^{3}$ School of Computer Engineering, Jiangsu University of Technology, Changzhou 213001, China \\ Correspondence should be addressed to Naibao He; henaibao@126.com
}

Received 19 April 2015; Revised 8 July 2015; Accepted 22 July 2015

Academic Editor: Carlo Cosentino

Copyright (C) 2015 Naibao He et al. This is an open access article distributed under the Creative Commons Attribution License, which permits unrestricted use, distribution, and reproduction in any medium, provided the original work is properly cited.

\begin{abstract}
A novel flight control scheme is proposed using the terminal sliding mode technique, unidirectional auxiliary surfaces and the disturbance observer model. These proposed dynamic attitude control systems can improve control performance of hypersonic vehicles despite uncertainties and external disturbances. The terminal attractor is employed to improve the convergence rate associated with the critical damping characteristics problem noted in short-period motions of hypersonic vehicles. The proposed robust attitude control scheme uses a dynamic terminal sliding mode with unidirectional auxiliary surfaces. The nonlinear disturbance observer is designed to estimate system uncertainties and external disturbances. The output of the disturbance observer aids the robust adaptive control scheme and improves robust attitude control performance. Finally, simulation results are presented to illustrate the effectiveness of the proposed terminal sliding mode with unidirectional auxiliary surfaces.
\end{abstract}

\section{Introduction}

It is well known that the near space hypersonic vehicle (NHV) as a novel aerospace aircraft plays an important role in military and civil applications [1]. Compared with traditional flight vehicles, the NHV has significant difference in flight airspace, working states, and mission patterns. In addition, it possesses many advantages over traditional spacecraft, such as lower launch cost, maintainability, speed of redeployment, and reusability. Consequently, the NHV concept has garnered considerable interest over the last few years. On the other hand, the NHV dynamics is severely nonlinear, timevarying, highly uncertain, and strongly coupled. It also suffers different external disturbances with changes in the flight environment, propulsion system perturbation, atmospheric disturbances, and other factors. Therefore, robust flight control of the NHV is a challenging problem. Many advanced control schemes have been presented in the literatures for control of NHVs. For example, Chen and Jiang [2] developed robust attitude control for NHVs with time-varying disturbances. Zhao et al. [3, 4] proposed a fault-tolerant control scheme based on a second-order dynamic terminal sliding mode. Their model calls for a near space vehicle (NSV) attitude dynamic system in the presence of model parameter uncertainties and external disturbances. He et al. [5] designed an adaptive output feedback fault-tolerant controller for the longitudinal dynamics of a generic hypersonic flight vehicle. The robust sliding mode control strategy was applied to the NSV attitude dynamics. In [6], an adaptive nonsingular terminal sliding mode approach was presented for the attitude control of NHV in the presence of parameter uncertainties and external disturbances. In [7], a second-order dynamic terminal sliding mode control was proposed for the NSV with high speed, violent changes of aerodynamic parameters, and large external disturbance. The adaptive attitude control was studied for the NSV with dynamic uncertainties using a functional link network (FLN) in [8]. In $[9,10]$, an adaptive tracking control was proposed for the NSV using TakagiSugeno fuzzy models. In [11, 12], a robust back-stepping control was studied for a flexible air-breathing hypersonic 
vehicle in the presence of input constraint and aerodynamic uncertainty. The disturbance observer-based control method was proposed for uncertain flight dynamics system in [13-16]. In the above-mentioned control approaches, sliding mode control (SMC) has been extensively studied for handling systems with large uncertainties, nonlinearities, and bounded external disturbances. Due to remarkable robust control performance, the SMC has been widely applied in many practical systems [17-23].

In order to alleviate chattering, a new approach called sliding mode control with unidirectional auxiliary surfaces (UAS-SMC) has been proposed to constrain the system states [24-27]. The unidirectional auxiliary surfaces in UAS-SMC are used to construct a positive invariant set. It implies that once the system states come into this positive invariant set, they would never come out again. However, the UAS-SMC scheme has a specific disadvantage. Its drawback is that the convergence speed of UAS-SMC is slower than that of the conventional SMC. This drawback prevents the UAS-SMC strategy from being applied to some high-frequency systems such as the angular rate loop of flight control systems.

Based on the above analysis, the key breakthroughs can be concluded as follows: The aim of this paper is to first propose UAS-TSMC with suitably high convergence speed. The linear switching surface in UAS-SMC strategy is replaced by a terminal switching surface in UAS-TSMC to improve the convergence speed. And the nonlinear unidirectional auxiliary surfaces in UAS-TSMC can be proven to stay in a positive invariant set. Secondly, by combining the nonlinear disturbance observer with the adaptive terminal sliding control via unidirectional auxiliary surfaces (UAS-TSMC-NDO), a composite UAS-TSMC-NDO method is constructed to handle the external disturbances, and it can make the system more stable, alleviate chattering, and strengthen robustness.

This work is motivated by the above discussion. A novel UAS-TSMC-NDO control scheme is proposed for the NHV, which has been applied to the tracking control of attitude angles. The organization of the paper is as follows: Section 2 details the problem statement, and Section 3 gives the design of terminal sliding mode control with unidirectional auxiliary surfaces based on the disturbance observer. Simulation results are presented to demonstrate the effectiveness of our proposed approaches given in Section 4, followed by the conclusion remarks in Section 5.

\section{Problem Statement}

The attitude mathematic model for hypersonic vehicles developed at NASA Langley Research Center is given by [28]

$$
\begin{aligned}
\dot{\alpha} & =\frac{1}{M V \cos \beta}+\left[-L+M g \cos \gamma \cos \mu-T_{x} \sin \alpha\right. \\
& \left.+T_{z} \cos \alpha\right]+q-\tan \beta(p \cos \alpha+r \sin \alpha), \\
\dot{\beta} & =\frac{1}{M V}\left[-T_{x} \sin \beta \cos \alpha+T_{y} \cos \beta-T_{z} \sin \alpha \sin \beta\right] \\
& +\frac{1}{M V}[Y \cos \beta+M g \cos \gamma \sin \mu]-r \cos \alpha+p \sin \alpha,
\end{aligned}
$$

$$
\begin{aligned}
\dot{\mu}= & \sec \beta(p \cos \alpha+r \sin \alpha)+\frac{1}{M V}[L \tan \gamma \sin \mu \\
& +L \tan \beta]+\frac{1}{M V}[-M g \cos \gamma \cos \mu \tan \beta \\
& \left.+\left(Y+T_{y}\right) \cos \beta \cos \mu \tan \gamma\right] \\
& +\frac{1}{M V}\left[\left(T_{x} \sin \alpha-T_{z} \cos \alpha\right)(\tan \gamma \sin \mu+\tan \beta)\right] \\
& -\frac{1}{M V}\left[\left(T_{x} \cos \alpha+T_{z} \sin \alpha\right) \tan \gamma \cos \mu \sin \beta\right], \\
\dot{p}= & I_{q r}^{p} q r+\dot{I}_{p}^{p} p+g_{l}^{p}\left(l_{A}+l_{T}\right), \\
\dot{q}= & I_{p r}^{q} p r+\dot{I}_{q}^{q} q+g_{m}^{q}\left(m_{A}+m_{T}\right), \\
\dot{r}= & I_{p q}^{r} p q+\dot{I}_{r}^{r} r+g_{n}^{r}\left(n_{A}+n_{T}\right),
\end{aligned}
$$

where $\alpha$ is the angle of attack, $\beta$ is the sideslip angle, $\mu$ is the bank angle, $p$ is the roll rate, $q$ is the pitch rate, and $r$ is the yaw rate.

According to the singular perturbation theory, the six equations can be divided into the fast loop and the slow loop, respectively. In order to study robust attitude control for the NHV, the kinematic and dynamic attitudes of the hypersonic vehicle are described as the following multi-input and multioutput (MIMO) affine nonlinear system [29]:

$$
\begin{aligned}
& \dot{x}_{1}=f_{1}\left(x_{1}\right)+g_{1}\left(x_{1}\right) x_{2}, \\
& \dot{x}_{2}=f_{2}\left(x_{1}, x_{2}\right)+g_{2}\left(x_{1}, x_{2}\right) u+\psi(t), \\
& y=x_{1},
\end{aligned}
$$

where $x_{1}=[\alpha, \beta, \mu]^{T}$ is the slow loop state, the attitude angle vector. $x_{2}=[p, q, r]^{T}$ is the fast loop state, the bodyaxis angular rate vector. $\psi(t)$ is the compounded external disturbance. $u$ is the system control input and $y$ is the system output. The detailed expressions of $f_{1}\left(x_{1}\right), f_{2}\left(x_{1}, x_{2}\right), g_{1}\left(x_{1}\right)$, and $g_{2}\left(x_{1}, x_{2}\right)$ can be found in [29].

In this paper, the attitude control objective is that the robust control schemes are designed to track a given desired output $y_{d}$ of the attitude motion. In the attitude control design, we define

$$
\begin{aligned}
& e_{1}=y-y_{d}, \\
& e_{2}=x_{2}-\pi_{1},
\end{aligned}
$$

where $e_{1}$ is the attitude angle tracking error vector, $e_{2}$ is the attitude angular rate tracking error vector, and $\pi_{1} \in R^{3}$ is a command signal which will be designed.

In order to proceed with the design of robust attitude control scheme for attitude motions (2a), (2b), and (2c) of the $\mathrm{NHV}$, the following assumptions are required.

Assumption 1. For the compounded disturbance $\psi(t)$, there exists an unknown positive constant $\vartheta$ such that $\|\dot{\psi}(t)\| \leq \vartheta$. 
Assumption 2. For all $t>0$, there exist $\Upsilon_{i}$ such that $\left\|y_{d}^{(i)}(t)\right\| \leq$ $\Upsilon_{i}, i=1,2$.

\section{Design of Terminal Sliding Mode Control with Unidirectional Auxiliary Surfaces}

In this section, a terminal sliding mode control with unidirectional auxiliary is developed. First, a terminal sliding mode control algorithm is designedto stabilize the slow loop system and ensure that the unidirectional auxiliary sliding mode converges to the equilibrium surfaces asymptotically. Then, the robust adaptive control will be designed based on disturbance observer that guarantees the fast loop system will remain stable in the presence of uncertainties and external disturbances effecting the NHV.

3.1. Slow Loop Terminal Sliding Mode Controller Design. Considering the slow loop dynamic (3) of the NHV and differentiating $e_{1}$ yielded

$$
\begin{aligned}
\dot{e}_{1} & =\dot{y}-\dot{y}_{d}=f_{1}\left(x_{1}\right)+g_{1}\left(x_{1}\right) x_{2}-\dot{y}_{d} \\
& =f_{1}\left(x_{1}\right)+g_{1}\left(x_{1}\right) e_{2}-\dot{y}_{d}+g_{1}\left(x_{1}\right) \pi_{1} .
\end{aligned}
$$

The detailed design of UAS-TSMC is given as follows.

Step 1. The terminal switching surfaces for system (5) are given by

$$
\begin{aligned}
& S_{1 i}=e_{1 i}+\xi_{12 i} \cdot\left(\int e_{1 i}+\xi_{3 i} \int e_{1 i}^{n / m}\right)=0, \\
& S_{2 i}=e_{1 i}+\xi_{22 i} \cdot\left(\int e_{1 i}+\xi_{3 i} \int e_{1 i}^{n / m}\right)=0,
\end{aligned}
$$

where $\xi_{12 i}>\xi_{22 i}>0, \xi_{3 i}>0$ are the designed constants and $m$ and $n$ are positive odd integers, which satisfy the following condition:

$$
m>n \text {. }
$$

Defining $z=\left[z_{2 i}, z_{1 i}\right]^{T}, z_{2 i}=e_{1 i}$, and $z_{1 i}=\int e_{1 i}+\xi_{3 i} \int e_{1 i}{ }^{n / m}$, the terminal switching surfaces in (6) can be rewritten as

$$
\begin{aligned}
& S_{1 i}=z_{2 i}+\xi_{12 i} \cdot z_{1 i}=0, \\
& S_{2 i}=z_{2 i}+\xi_{22 i} \cdot z_{1 i}=0 .
\end{aligned}
$$

Step 2. Based on the terminal switching surface $S_{1 i}, S_{2 i}$, number $0 i, \ldots, 3 i$ subspace can be defined as in Figure 1.

As shown in Figure 1, the appropriate points $P_{S 1 i^{+}}, P_{S 1 i^{-}}$, $P_{S 2 i+}$, and $P_{S 2 i-}$ should be selected on terminal switching surfaces $S_{1 i}, S_{2 i}$, where points $P_{S 1 i+}, P_{S 2 i-}$ are located in the fourth quadrant and points $P_{S 1 i-}, P_{S 2 i+}$ are located in the second quadrant. The lines $P_{S 1 i} P_{S 2 i-}, P_{S 1 i+} P_{S 2 i-}, P_{S 1 i-} P_{S 2 i+}$, and $P_{S 1 i+} P_{S 2 i+}$ defined in Figure 2 are UAS $H_{0 i}, H_{1 i}, H_{2 i}$, and $H_{3 i}$, respectively. For the simplicity of discussion, we define that the UAS $H_{k i}, k \in\{0,1,2,3\}$, is designed by points $P_{S 1 i}$, $P_{S 2 i}$ where $P_{S 1 i} \in\left\{P_{S 1 i+}, P_{S 1 i-}\right\}, P_{S 2 i} \in\left\{P_{S 2 i+}, P_{S 2 i-}\right\}$. The expression of UAS $H_{k i}$ is given as follows:

$$
H_{k i}(z)=\omega_{k i 1} \cdot z_{2 i}+\omega_{k i 2} \cdot z_{1 i}+M_{i},
$$

where $H_{k i}\left(P_{S 1 i}\right)=0, H_{k i}\left(P_{S 2 i}\right)=0, \omega_{k i 1} \neq 0, M_{i}>0$.

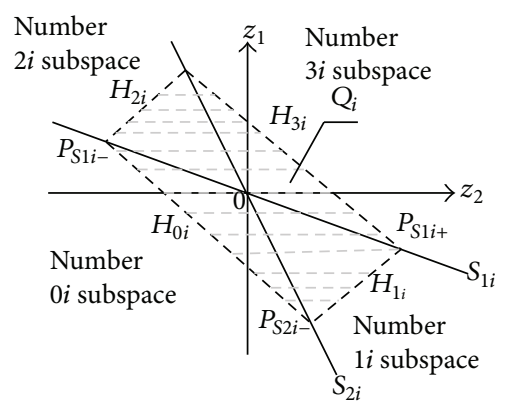

Figure 1: The UAS $H_{0 i}, H_{1 i}, H_{2 i}$, and $H_{3 i}$.

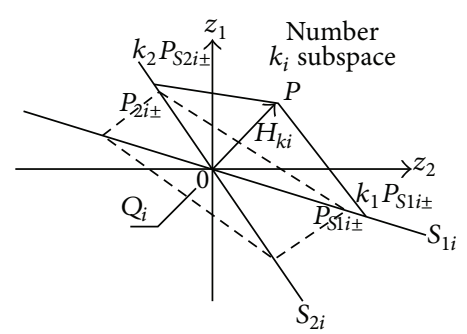

Figure 2: The point $p$ in number $k_{i}$ subspace.

Remark 3. These UAS $H_{0 i}, H_{1 i}, H_{2 i}, H_{3 i}$ should constitute a convex quadrilateral which is given by

$$
Q_{i}=\left\{\left(z_{2 i}, z_{1 i}\right) \mid H_{k i}(z) \geq 0, k=0,1,2,3\right\} .
$$

Hence, the system states $e_{i}$ in set $Q_{i}$ imply that the system states satisfy the state constraints. This convex quadrilateral can be proved to be a positively invariant set [30] with control equation (9).

Step 3. The UAS in Figure 1 would be used to design control input $\pi_{1}$ when the states are moving in number $0_{i}, \ldots, 3_{i}$ subspace. The compact form of current UAS $H_{i}$ is given by

$$
H_{i}(z)=\Omega_{i 1} \cdot z_{2 i}+\Omega_{i 2} \cdot z_{1 i}+M_{i},
$$

where

$$
\begin{gathered}
\Omega_{i 1}= \begin{cases}\omega_{0 i 1} & S_{1 i}<0, S_{2 i}<0 \\
\omega_{1 i 1} & S_{1 i}<0, S_{2 i} \geq 0 \\
\omega_{2 i 1} & S_{1 i} \geq 0, S_{2 i}<0 \\
\omega_{3 i 1} & S_{1 i} \geq 0, S_{2 i} \geq 0,\end{cases} \\
\Omega_{i 2}= \begin{cases}\omega_{0 i 2} & S_{1 i}<0, S_{2 i}<0 \\
\omega_{1 i 2} & S_{1 i}<0, S_{2 i} \geq 0 \\
\omega_{2 i 2} & S_{1 i} \geq 0, S_{2 i}<0 \\
\omega_{3 i 2} & S_{1 i} \geq 0, S_{2 i} \geq 0 .\end{cases}
\end{gathered}
$$


Step 4. The UAS-TSMC control law for system (5) is designed as

$$
\begin{gathered}
\pi_{1}=g_{1}\left(x_{1}\right)^{-1}\left(-f_{1}\left(x_{1}\right)-\Omega_{i 1}^{-1} \cdot \Omega_{i 2} \cdot e_{1}-\xi_{3 i} \Omega_{i 1}^{-1}\right. \\
\left.\cdot \Omega_{i 2} \cdot e_{1}^{n / m}+\dot{y}_{d}-g_{1}\left(x_{1}\right) \cdot e_{2}+\Omega_{i 1}^{-1} \cdot N\right)
\end{gathered}
$$

where $N>\sup \left\{-\Omega_{1 i} \cdot \psi\right\}$ is the approaching law.

Remark 4. $\pi_{1}$ is a nonsingular sliding mode surface, which does not produce singularities. But it may cause singularity when we compute the time derivative of $\pi_{1}$. Therefore, in the design of the controller, we add the saturation function in order to overcome the singularity problem [31].

Definition 5 (see [30]). The set $Q_{i}$ is said to be positively invariant for system (5) with control law in (13). If for all $e_{1}(t) \in Q_{i}$ the solution $e_{1}(t) \in Q_{i}$ for $t>0$. If $e_{1}(0) \in Q_{i}$ implies $e_{1}(t) \in Q_{i}$ for all $t \in R$, we say that $Q_{i}$ is invariant.

Remark 6. As shown in Figure 2, the set $Q_{i}=\left\{\left(z_{2 i}, z_{1 i}\right) \mid\right.$ $\left.H_{k i}(z) \geq 0, k=0,1,2,3\right\}$ is a convex set. For the point $p(t)=$ $\left[z_{2 i}(t), z_{1 i}(t)\right]^{T} \notin Q_{i}$, if the point $p(t)$ locates in number $k_{i}$ subspace where $k \in\{0,1,2,3\}$, there exists $H_{k i}(p(t))<0$.

Lemma 7. For the point $p=\left[z_{1 i}, z_{2 i}\right]^{T}$ in number $k_{i}$ subspace where $k \in\{0,1,2,3\}$, the UAS $H_{k i}(\mathbf{z})$ in No. $k_{i}$ subspace is designed by points $p_{S 1 i}, p_{S 2 i}$ where $H_{k i}\left(p_{S 1 i}\right)=0$ and $H_{k i}\left(p_{S 2 i}\right)=0$. The expression of UAS $H_{k i}(\mathbf{z})$ is written as

$$
H_{k i}(\mathbf{z})=\omega_{k i 1} \cdot z_{2 i}+\omega_{k i 2} \cdot z_{1 i}+M_{i}
$$

where $M_{i}>0$. Then, for point $p$, there exists the fact that $M_{i}-$ $H_{k i}(p) \geq 0$ and $p=[0,0]^{T}$ when $H_{k i}(p)=M_{i}$.

Proof. Define the coordinates of points $p_{S 1 i}$ and $p_{S 2 i}$ as follows:

$$
\begin{aligned}
& p_{S 1 i}=\left[z_{12}, z_{11}\right]^{T}, \\
& p_{S 2 i}=\left[z_{22}, z_{21}\right]^{T} .
\end{aligned}
$$

As shown in Figure 2, it is noted that, for the point $p=$ $\left[z_{1 i}, z_{2 i}\right]^{T}$ in number $k_{i}$ subspace, there exists $p=k_{1} p_{s 1 i}+$ $k_{2} p_{s 2 i}, k_{1}, k_{2} \geq 0$. Then, the coordinate of point $p$ can be expressed as follows:

$$
p=\left[k_{1} \cdot z_{12}+k_{2} \cdot z_{22}, k_{1} \cdot z_{11}+k_{2} \cdot z_{21}\right]^{T} .
$$

In (14) with (16), one has

$$
\begin{aligned}
H_{k i}(p)= & \omega_{k i 1} \cdot\left(k_{1} \cdot z_{12}+k_{2} \cdot z_{22}\right)+M_{i}+\omega_{k i 2} \\
& \cdot\left(k_{1} \cdot z_{11}+k_{2} \cdot z_{21}\right) \\
= & k_{1} \cdot\left(\omega_{k i 1} \cdot z_{12}+\omega_{k i 2} \cdot z_{11}+M_{i}\right) \\
& -\left(k_{1}+k_{2}\right) \cdot M_{i}+k_{2} \\
& \cdot\left(\omega_{k i 1} \cdot z_{22}+\omega_{k i 2} \cdot z_{21}+M_{i}\right) .
\end{aligned}
$$

From the condition $H_{k i}\left(p_{S 1 i}\right)=0, H_{k i}\left(p_{S 2 i}\right)=0$, we have

$$
\begin{aligned}
& H_{k i}\left(p_{S 1 \mathrm{i}}\right)=\omega_{k i 1} \cdot z_{12}+\omega_{k i 2} z_{11}+M_{i}=0, \\
& H_{k i}\left(p_{S 2 \mathrm{i}}\right)=\omega_{k i 1} \cdot z_{22}+\omega_{k i 2} z_{21}+M_{i}=0 .
\end{aligned}
$$

Substituting (18) into (17), we obtain

$$
H_{k i}(p)=-\left(k_{1}+k_{2}\right) M_{i}+M_{i}
$$

Hence, the first conclusion is as follows: $M_{i}-H_{k i}(p)=\left(k_{1}+\right.$ $\left.k_{2}\right) \cdot M_{i} \geq 0$.

When $H_{k i}(p)=M_{i}$, there exists $-\left(k_{1}+k_{2}\right) M_{i}=0$ from (19). Since $k_{1}, k_{2} \geq 0$ and $-\left(k_{1}+k_{2}\right) M_{i}=0$, we can conclude $k_{1}=0, k_{2}=0$. Then, we can obtain the second conclusion as follows:

$$
\begin{aligned}
p & =\left[z_{2 i}, z_{1 i}\right]^{T} \\
& =\left[k_{1} \cdot z_{12}+k_{2} \cdot z_{22}, k_{1} \cdot z_{11}+k_{2} \cdot z_{21}\right]^{T}=[0,0]^{T} .
\end{aligned}
$$

This completes the proof.

Theorem 8. Considering system (5) with the control law in (13), system (5) is asymptotically stable. And the set $Q_{i}=$ $\left\{\left(z_{2 i}, z_{1 i}\right) \mid H_{k i}(\mathbf{z}) \geq 0, k=0,1,2,3\right\}$ can be guaranteed as a positively invariant set [30].

Proof. See the Appendix.

3.2. Fast Loop Terminal Sliding Mode Disturbance Observer Design. In this section, a robust flight control approach will be developed based on the combination of nonlinear disturbance observer and terminal sliding mode control with unidirectional auxiliary surfaces. For control of external disturbances and model uncertainties in the fast loop, they cannot be directly used to design the robust sliding mode controller. To efficiently handle them, the nonlinear disturbance observer is proposed to estimate them.

In order to estimate the external disturbance $\psi$ of the near space hypersonic vehicle system, the general nonlinear near space hypersonic vehicle fast loop dynamic error model in (4) will be used. Considering (4) and differentiating $e_{2}$ yielded

$$
\begin{aligned}
\dot{e}_{2} & =\dot{x}_{2}-\dot{\pi}_{1} \\
& =f_{2}\left(x_{1}, x_{2}\right)+g_{2}\left(x_{1}, x_{2}\right) u+\psi(t)-\dot{\pi}_{1} .
\end{aligned}
$$

The disturbance observer is proposed as follows [32]:

$$
\begin{aligned}
\widehat{\psi}= & z+P\left(e_{2}\right), \\
\dot{z}= & -L\left(e_{2}\right) z \\
& -L\left(e_{2}\right)\left(P\left(e_{2}\right)+f\left(x_{1}, x_{2}\right)+g\left(x_{1}, x_{2}\right) u\right),
\end{aligned}
$$

where $z$ is an auxiliary variable of the proposed disturbance observer, $\widehat{\psi}$ is the output of the nonlinear disturbance 
observer, $P\left(e_{2}\right)=\left[P_{1}\left(e_{2}\right), P_{2}\left(e_{2}\right), \ldots, P_{n}\left(e_{2}\right)\right] \in R^{n}$, and $L\left(e_{2}\right)$ is an appropriate dimension gain matrix which is given by

$$
\begin{aligned}
L\left(e_{2}\right)= & \frac{\partial P\left(e_{2}\right)}{\partial e_{2}} \\
= & {\left[\begin{array}{cccc}
\frac{\partial P_{1}\left(e_{2}\right)}{\partial e_{21}} & \frac{\partial P_{1}\left(e_{2}\right)}{\partial e_{22}} & \cdots & \frac{\partial P_{1}\left(e_{2}\right)}{\partial e_{2 n}} \\
\frac{\partial P_{2}\left(e_{2}\right)}{\partial e_{21}} & \frac{\partial P_{2}\left(e_{2}\right)}{\partial e_{22}} & \cdots & \frac{\partial P_{2}\left(e_{2}\right)}{\partial e_{2 n}} \\
\vdots & \vdots & \ddots & \vdots \\
\frac{\partial P_{n}\left(e_{2}\right)}{\partial e_{21}} & \frac{\partial P_{n}\left(e_{2}\right)}{\partial e_{22}} & \cdots & \frac{\partial P_{n}\left(e_{2}\right)}{\partial e_{2 n}}
\end{array}\right] . }
\end{aligned}
$$

Define the estimate error $e=\psi-\widehat{\psi}$. Since there is no prior information about the derivative of the disturbance $\psi$, it is reasonable to suppose that $\dot{\psi}=0$, which implies that the disturbance varies slowly relative to the observer dynamics.

Differentiating $e$, we have

$$
\dot{e}=\dot{\psi}-\dot{\widehat{\psi}} \approx-\dot{\hat{\psi}}
$$

Considering (18) and differentiating with respect to time yielded

$$
\begin{aligned}
\dot{\hat{\psi}}= & \dot{z}+L\left(e_{2}\right) \dot{x}_{2} \\
= & -L\left(e_{2}\right) z \\
& -L\left(e_{2}\right)\left(P\left(e_{2}\right)+f\left(x_{1}, x_{2}\right)+g\left(x_{1}, x_{2}\right) u\right) \\
& +L\left(e_{2}\right) \dot{x}_{2}=L\left(e_{2}\right)(\psi-\widehat{\psi})=L\left(e_{2}\right) e .
\end{aligned}
$$

Thus, we have $\dot{e}=-L\left(e_{2}\right) e$. It is apparent that the choice of $L\left(e_{2}\right)$ must make the disturbance observer error converge to zero.

According to the design of terminal sliding mode with unidirectional auxiliary surfaces, the robust adaptive control of the hypersonic vehicle based on estimated disturbance observation can be surmised in the following theorem.

Theorem 9. For NHV dynamic error model (4), the disturbance observer is designed as (22), and there exists a terminal sliding mode with unidirectional auxiliary surfaces (26), under the two terminal switching surfaces (28), (29), the adaptive law (27) and the NDO-based robust flight control law (30). Then the robust stability of the control system in the presence of the parameter uncertainties and external disturbances is guaranteed:

$$
\begin{aligned}
& H=\Omega_{1} e_{2}+\Omega_{2}\left(\int e_{2}+\int e_{2}^{n / m}\right)+M, \\
& \dot{\hat{\eta}}=\lambda \delta, \\
& S_{1}=e_{2}+\xi_{1}\left(\int e_{2}+\int e_{2}^{n / m}\right),
\end{aligned}
$$

$$
\begin{aligned}
S_{2}= & e_{2}+\xi_{2}\left(\int e_{2}+\int e_{2}^{n / m}\right), \\
u= & g_{2}\left(x_{1}, x_{2}\right)^{-1}\left(-f_{2}\left(x_{1}, x_{2}\right)\right) \\
& +\Omega_{1}^{-1}\left(-\Omega_{2} \cdot e_{2}^{n / m}+\lambda \delta+\widehat{\eta}+\dot{\pi}_{1}\right), \\
\delta= & \Omega_{1} e_{2}+\Omega_{2}\left(\int e_{2}+\int e_{2}^{n / m}\right),
\end{aligned}
$$

where, $-\Omega_{1} \psi \leq \eta, \eta>0, M>0, \lambda>0$ are designed constants and $m$ and $n$ are positive odd integers and satisfy $1<m / n<2$.

Proof. Consider the following Lyapunov function:

$$
V=\frac{1}{2} \delta^{T} \delta+\frac{1}{2 \lambda} \widetilde{\eta}^{T} \widetilde{\eta}
$$

Differentiating $V$ with respect to time yields

$$
\begin{aligned}
\dot{V} & =-\delta^{T} \dot{\delta}-\frac{1}{\lambda} \widetilde{\eta}^{T} \dot{\bar{\eta}}=-\delta^{T}\left(\Omega_{1} \dot{e}_{2}+\Omega_{2} e_{2}+\Omega_{2} e_{2}{ }^{n / m}\right) \\
& -\frac{1}{\lambda} \widetilde{\eta}^{T} \dot{\hat{\eta}}=-\delta^{T}\left(\Omega_{1} \dot{e}_{2}+\Omega_{2} e_{2}+\Omega_{2} e_{2}{ }^{n / m}\right)-\frac{1}{\lambda} \widetilde{\eta}^{T} \dot{\hat{\eta}} \\
& =-\delta^{T}\left[\Omega _ { 1 } \left(\Omega _ { 1 } ^ { - 1 } \left(-\Omega_{2} e_{2}-\Omega_{2} e_{2}^{n / m}+\lambda \delta+\widehat{\eta}\right.\right.\right. \\
& \left.\left.\left.+\dot{\pi}_{1}\right)+\psi-\dot{\pi}_{1}\right)+\Omega_{2} e_{2}+\Omega_{2} e_{2}^{n / m}\right]-\widetilde{\eta}^{T} \delta \\
& \leq-\delta^{T} \lambda \delta-\delta^{T} \widehat{\eta}-\delta^{T} \Omega_{1} \psi-\delta^{T} \widetilde{\eta}=-\delta^{T} \lambda \delta-\delta^{T}(\widehat{\eta} \\
& \left.+\Omega_{1} \psi+\widetilde{\eta}\right) \leq-\delta^{T} \lambda \delta-\delta^{T}(\widehat{\eta}-\eta+\widetilde{\eta}) \\
& \leq \lambda_{1 \mathrm{~min}}\|\delta\|^{2}<0 .
\end{aligned}
$$

Thus, the proof is completed.

\section{Simulation Study}

To illustrate the effectiveness of the proposed robust control scheme, the simulations are given for the near space hypersonic vehicles. The initial conditions are chosen as follows: $M=13680 \mathrm{~kg}, V(0)=3000 \mathrm{~m} / \mathrm{s}, X(0)=1000 \mathrm{~m}, Y(0)=$ $1000 \mathrm{~m}, Z(0)=-30 \mathrm{~km}, m=9, n=7$, the attitude angle $\alpha(0)=0 \mathrm{deg}, \beta(0)=0 \mathrm{deg}, \gamma(0)=0 \mathrm{deg}$, the attitude angle rate $p(0)=0 \mathrm{deg} / \mathrm{s}, q(0)=3 \mathrm{deg} / \mathrm{s}$, and $r(0)=0 \mathrm{deg} / \mathrm{s}$. In order to guarantee the stability of the near space hypersonic vehicles attitude, the reference signals of the attitude angle are chosen as $\alpha_{c}=4 \mathrm{deg}, \beta_{c}=0 \mathrm{deg}$, and $\gamma_{c}=-2 \mathrm{deg}$. The disturbances are considered as follows:

$$
\begin{aligned}
& d_{1}(t)=200000 \sin (\pi t) \mathrm{Nm}, \\
& d_{2}(t)=5000000 \sin (\pi t) \mathrm{Nm}, \\
& d_{3}(t)=1000000 \sin (\pi t) \mathrm{Nm} .
\end{aligned}
$$

For the purpose of comparison for the effectiveness of TSMC between the proposed approach presented in this paper and an existing method, the corresponding simulation 

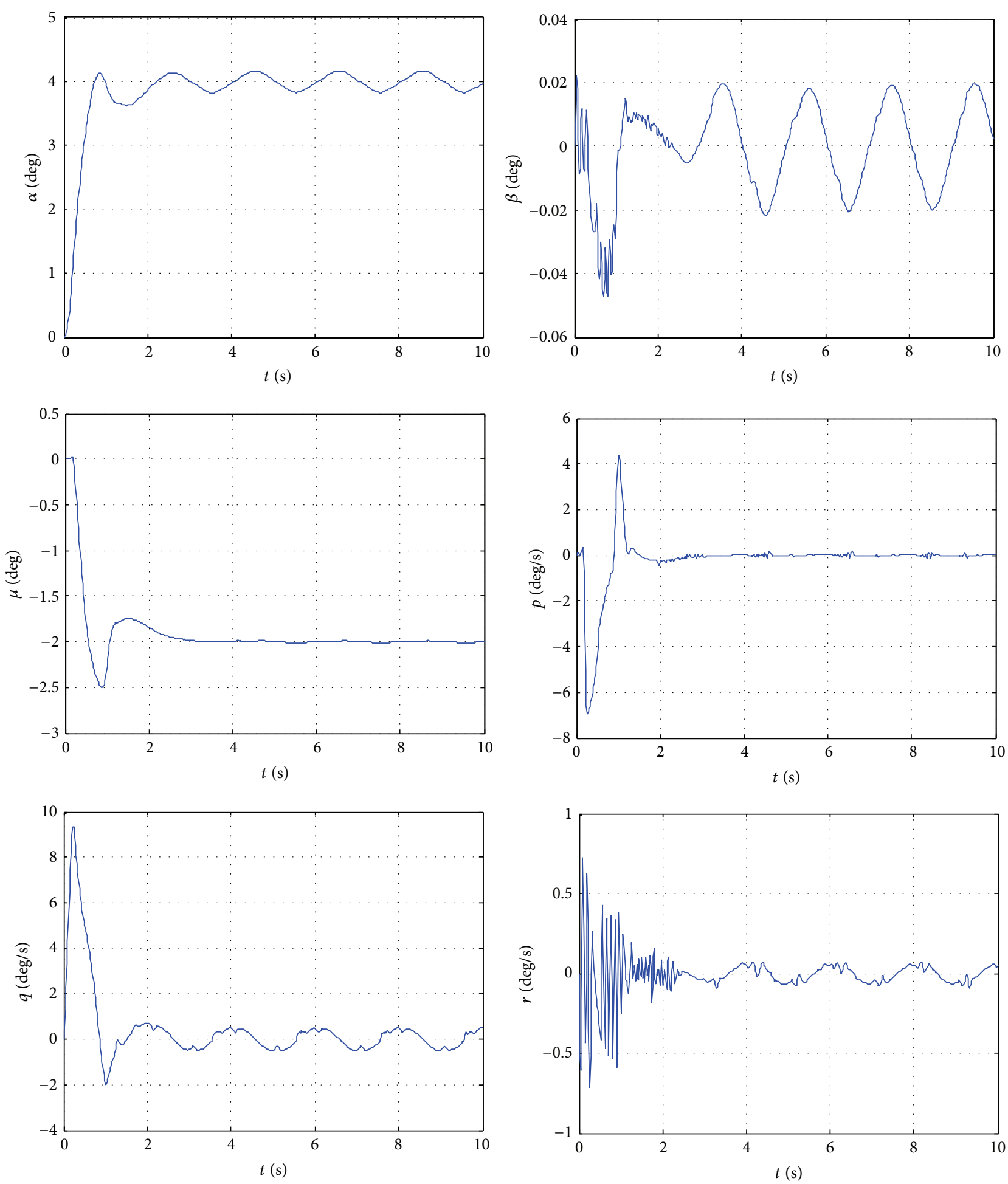

FIgURE 3: Simulation results with TSMC.

results are depicted in Figures 3-5. From Figure 3, it is shown that control torques responses using TSMC method cannot meet system performance requirements. From Figure 4, under the designed UAS-TSMC attitude control scheme, attitude angle rate and attitude angle become unstable quickly. The result shows that there exists seriously error during most of the simulation time. Furthermore, in Figure 5, the UAS-TSMC with NDO algorithm can make the system 

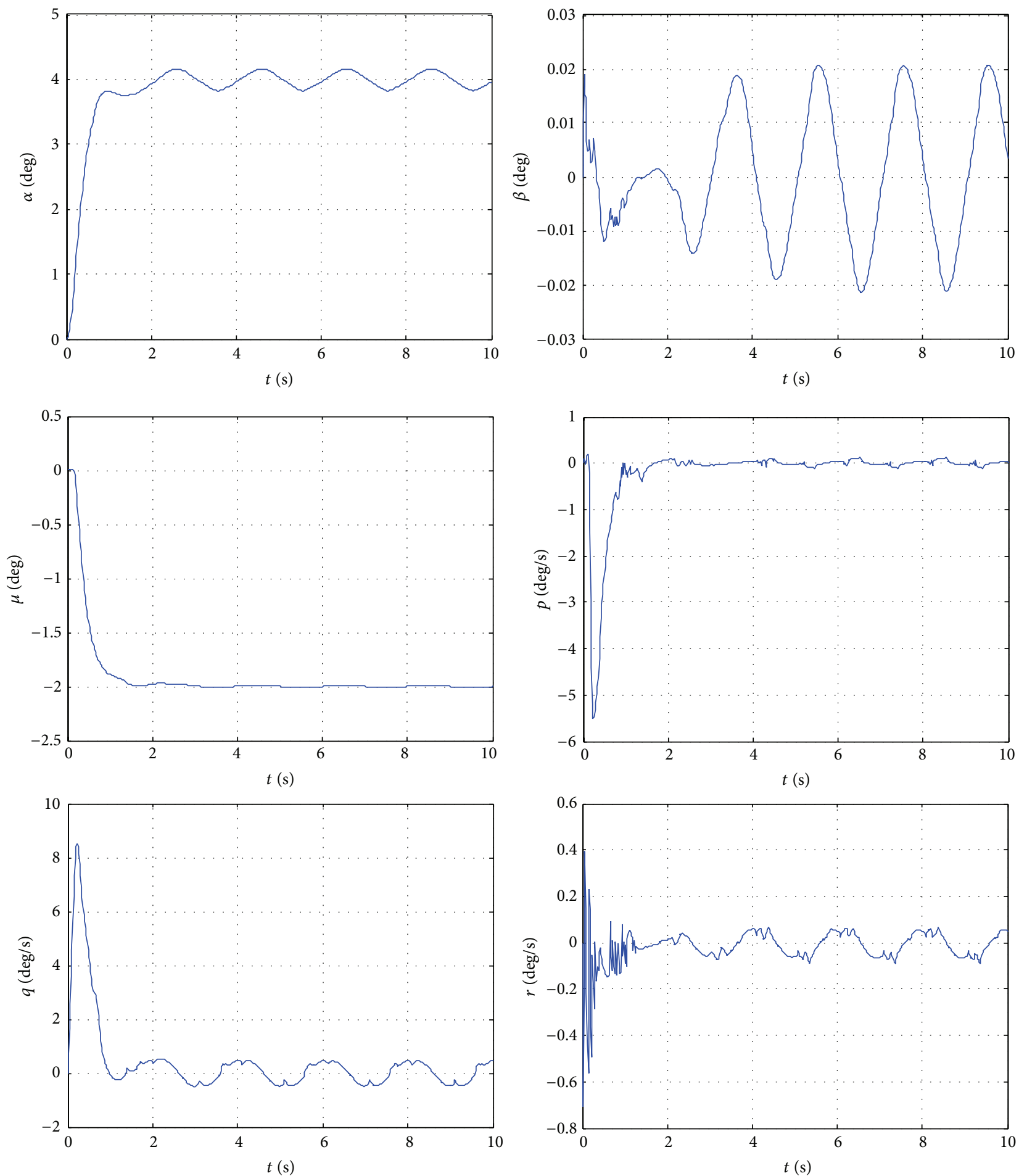

FIGURE 4: Simulation results with UAS-TSMC.

stable and indicates that all of the responses of attitude angle and angular velocity can track the desired objectives well. Thus, the robust flight control approach based on the UAS-TSMC with NDO can satisfy precision and robustness requirements in the presence of uncertainties and large external disturbances.

\section{Conclusion}

In this paper, a novel robust sliding mode control with unidirectional auxiliary surface approach has been developed to solve the tracking control problem for the NHV with functional uncertainty and external disturbance. UAS-TSMC 

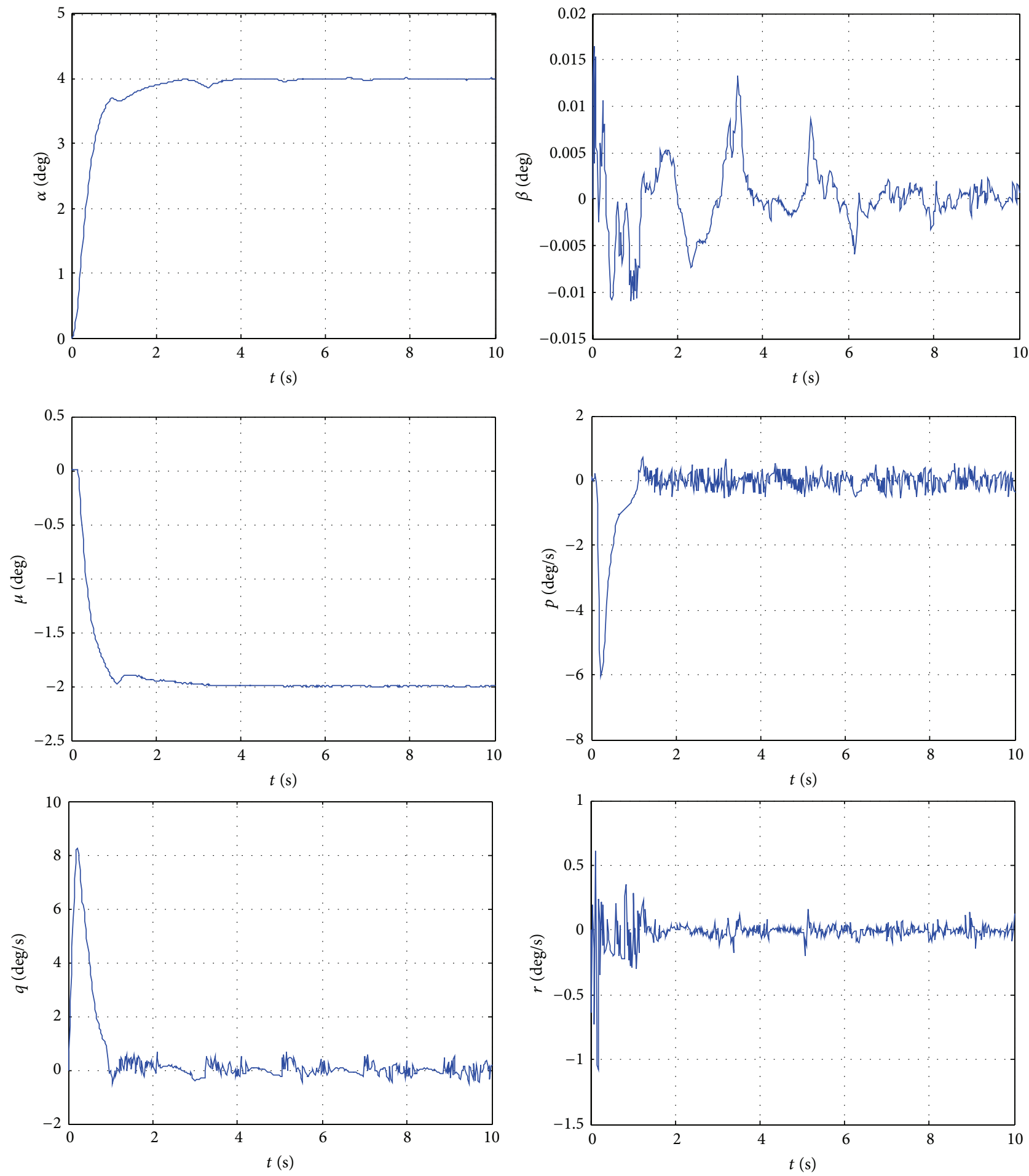

FIGURE 5: Simulation results with UAS-TSMC-NDO.

is employed to increase the robustness of the control system and improves the control performance. Meanwhile, the nonlinear disturbance observer has been developed to estimate uncertainties and external disturbances. Finally, the proposed method has been applied to near space hypersonic vehicles attitude dynamics, and simulation results have been given to illustrate the effectiveness of the proposed control approach. Studying attitude tracking control and accommodation approaches for NHVs with functional uncertainty and external disturbance will be investigated in our future work. 


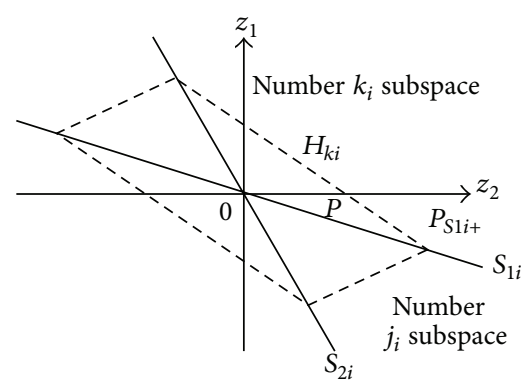

FIgURE 6: The point $\mathbf{z}$ between number $k_{i}$ and $j_{i}$ subspaces.

\section{Appendix}

Proof of Theorem 8. (1) The proof of the asymptotical stability for system (5) is given in this part.

For system (5), we consider the following Lyapunov function candidate:

$$
V_{i}(z)=\frac{1}{2} \cdot\left[M_{i}-\frac{H_{i}(z)}{M_{i}}\right]^{2} .
$$

Firstly, it would be proved that $V_{i} \geq 0$ and there exists $z=$ $\left[z_{2 i}, z_{1 i}\right]^{T}=[0,0]^{T}$ when $V_{i}=0$. From the definition of $V_{i}(\cdot)$, it follows that $V_{i}(\cdot) \geq 0$ and there exists $H_{i}(z)=M_{i}$ when $V_{i}=0$. Based on the discussion given in Lemma 7 , it is noted that $H_{k i}(z)=H_{k}(z)$ when $\mathbf{z}$ is located in number $k_{i}$ subspace where $k \in\{0,1,2,3\}$. Then, the condition $H_{k i}(z)=M_{i}$ implies $z=\left[z_{2 i}, z_{1 i}\right]^{T}$. Hence, there exists $z=\left[z_{2 i}, z_{1 i}\right]^{T}=$ $[0,0]^{T}$ when $V_{i}=0$.

Secondly, the conclusion $\dot{V}_{i}<0$ when $z \neq[0,0]^{T}$ is given as follows.

It follows from (11) and (13) that the time derivative of $H_{i}(\mathbf{z})$ is given as

$$
\begin{aligned}
\dot{H}_{i}(\mathbf{z}) & =\Omega_{i 1} \cdot \dot{z}_{2 i}+\Omega_{i 2} \cdot \dot{z}_{1 i} \\
& =\Omega_{i 1} \cdot \dot{e}_{1 i}+\Omega_{i 2} \cdot\left(\dot{e}_{1 i}+\xi_{3 i} \cdot e_{1 i}{ }^{n / m}\right)=N .
\end{aligned}
$$

Since $N=\dot{H}_{i}(\mathbf{z})$, the derivative of $H_{i}(\mathbf{z})$ should be a positive value.

From the Lyapunov function $V_{i}(\cdot)$ in (A.1), the derivative of $V_{i}(\cdot)$ is expressed as

$$
\dot{V}_{i}=-\left(M_{i}-H_{i}(z)\right) \cdot \frac{\dot{H}_{i}(z)}{M_{i}^{2}} .
$$

Based on Lemma 7, the conclusion is obtained that $H_{k i}(\mathbf{z})=$ $H_{i}(\mathbf{z})$ when $\mathbf{z}$ is located in number $k_{i}$ subspace and $M_{i}-$ $H_{k i}(\mathbf{z}) \geq 0$. As $\mathbf{z}=[0,0]^{T}$ when $H_{k i}(\mathbf{z})=M_{i}$, it implies that $M_{i}-H_{k i}(\mathbf{z})>0$ when $\mathbf{z} \neq[0,0]^{T}$. Then, there exists the fact that $M_{i}-H_{k i}(\mathbf{z})>0$ when $\mathbf{z} \neq[0,0]^{T}$. From $M_{i}-H_{k i}(\mathbf{z})>0$ and the positive derivative of $H_{k i}(\mathbf{z})$, the derivative of $V_{i}(\cdot)$ should be a negative value when $\mathbf{z} \neq[0,0]^{T}$.

Thirdly, the proof for the continuity of Lyapunov function $V_{i}(\cdot)$ in (A.1) is given in this part.

As shown in Figure 6, the point $\mathbf{z}$ on switching surface is selected to show the continuity of Lyapunov function $V_{i}(\cdot)$ in
(A.1) when the point $\mathbf{z}$ is switched between number $k_{i}$ and $j_{i}$, $k, j \in\{0,1,2,3\}, k_{i} \neq j_{i}$ subspace.

The point $p_{S 1 i}$ is one of the points which is used to design the UAS $H_{k i}$ and $H_{j i}$. Define the coordinates of point $p_{S 1 i}$ as $p_{S 1 i}=\left[z_{12}, z_{11}\right]^{T}$. From (18), the expressions of UAS $H_{k i}$ and $H_{j i}$ can be given as follows:

$$
\begin{aligned}
& H_{k i}(z)=\omega_{k i 1} \cdot z_{2 i}+\omega_{k i 2} \cdot z_{1 i}+M_{i}, \\
& H_{j i}(z)=\omega_{j i 1} \cdot z_{2 i}+\omega_{j i 2} \cdot z_{1 i}+M_{i} .
\end{aligned}
$$

Since the point $p_{S 1 i}$ is located on the UAS $H_{k i}$ and $H_{j i}$, (A.5) can be written as

$$
\begin{aligned}
& H_{k i}\left(p_{S 1 i}\right)=\omega_{k i 1} \cdot z_{12}+\omega_{k i 2} \cdot z_{11}+M_{i}=0, \\
& H_{j i}\left(p_{S 1 i}\right)=\omega_{j i 1} \cdot z_{12}+\omega_{j i 2} \cdot z_{11}+M_{i}=0 .
\end{aligned}
$$

For the point $\mathbf{z}$ in number $k_{i}$ subspace, there exists $\mathbf{z}=k_{1}$. $p_{S 1 i}=\left[k_{1} \cdot z_{12}, k_{1} \cdot z_{11}\right]^{T}, k_{1} \geq 0$, as given in Figure 6. By substituting the coordinate of point $\mathbf{z}$ into the UAS $H_{k i}$, we have

$$
\begin{aligned}
H_{k i}(z)= & \omega_{k i 1} \cdot k_{1} \cdot z_{12}+\omega_{k i 2} \cdot k_{1} \cdot z_{11}+M_{i} \\
= & k_{1}\left(\omega_{k i 1} \cdot z_{12}+\omega_{k i 2} \cdot z_{11}+M_{i}\right)-k_{1} \cdot M_{i} \\
& +M_{i} .
\end{aligned}
$$

Clearly, there exists $H_{k i}(z)=-k_{1} \cdot M_{i}+M_{i}$ by considering (A.5) and (A.6).

Then, the Lyapunov function $V_{i}(\cdot)$ in number $k_{i}$ subspace can be rewritten as follows:

$$
\begin{aligned}
V_{k i}(z) & =\frac{1}{2} \cdot\left[\frac{\left(M_{i}-H_{i}(z)\right)}{M_{i}}\right]^{2} \\
& =\frac{1}{2} \cdot\left[\frac{\left(M_{i}-H_{k i}(z)\right)}{M_{i}}\right]^{2}=\frac{1}{2} \cdot\left(k_{1}\right)^{2} .
\end{aligned}
$$

For the point $\mathbf{z}$ in number $j_{i}$ subspace, there exists $\mathbf{z}=k_{1}$. $p_{S 1 i}=\left[k_{1} \cdot z_{12}, k_{1} \cdot z_{11}\right]^{T}, k_{1} \geq 0$, as given in Figure 6. By substituting the coordinate of point $\mathbf{z}$ into the UAS $H_{j i}$, it is obtained that

$$
\begin{aligned}
H_{j i}(z)= & \omega_{j i 1} \cdot k_{1} \cdot z_{12}+\omega_{j i 2} \cdot k_{1} \cdot z_{11}+M_{i} \\
= & k_{1}\left(\omega_{j i 1} \cdot z_{12}+\omega_{j i 2} \cdot z_{11}+M_{i}\right)-k_{1} \cdot M_{i} \\
& +M_{i} .
\end{aligned}
$$

Clearly, there exists $H_{j i}(z)=-k_{1} \cdot M_{i}+M_{i}$ by considering (A.5) and (A.8).

Then the Lyapunov function $V_{i}(\cdot)$ in number $j_{i}$ subspace can be rewritten as follows:

$$
\begin{aligned}
V_{j i}(z) & =\frac{1}{2} \cdot\left[\frac{\left(M_{i}-H_{i}(z)\right)}{M_{i}}\right]^{2} \\
& =\frac{1}{2} \cdot\left[\frac{\left(M_{i}-H_{j i}(z)\right)}{M_{i}}\right]^{2}=\frac{1}{2} \cdot\left(k_{1}\right)^{2} .
\end{aligned}
$$


Hence, when the point $\mathbf{z}$ is switched between number $k_{i}$ and $j_{i}$ subspaces, there exists $V_{k i}(\mathbf{z})=V_{j i}(\mathbf{z})$. Then, the continuity of Lyapunov function $V_{i}(\cdot)$ in (A.1) is proved.

From the above demonstration, it is noted that the Lyapunov function $V_{i}(\cdot)$ in (A.1) is continuous; $V_{i} \geq 0$ and there exists $\mathbf{z}=\left[z_{1 i}, z_{2 i}\right]^{T}=[0,0]^{T}$ when $V_{i}=0 ; \dot{V}_{i}<0$ when $\mathbf{z} \neq[0,0]^{T}$. Hence, system (5) with the control law in (13) is asymptotically stable and $\mathbf{z}=\left[z_{2 i}, z_{1 i}\right]^{T}$ converges to $\mathbf{z}=[0,0]^{T}$. And the state $\mathbf{z}=\left[z_{2 i}, z_{1 i}\right]^{T}$ is defined as

$$
\begin{aligned}
& z_{2 i}=e_{1 i}, \\
& z_{1 i}=\int e_{1 i}+\xi_{3 i} \int e_{1 i}^{n / m} .
\end{aligned}
$$

It is clear that $\left[e_{2 i}, e_{1 i}\right]^{T}=[0,0]^{T}$ when $\mathbf{z}=\left[z_{2 i}, z_{1 i}\right]^{T}=$ $[0,0]^{T}$. Thus, we obtain that system (5) converges to $\mathbf{z}=$ $[0,0]^{T}$.

(2) Demonstration for the positively invariant set $Q_{i}=$ $\left\{\left(z_{2 i}, z_{1 i}\right) \mid H_{k i}(z)>0, k_{i}=0,1,2,3\right\}$ is given in this part. This conclusion is supported with the reduction to absurdity.

Considering the initial point $\mathbf{z}(0)=\left[z_{2 i}(0), z_{1 i}(0)\right]^{T} \in Q_{i}$ in number $k_{i}$ subspace, we suppose that there exists $\mathbf{z}\left(t_{1}\right)=$ $\left[z_{2 i}\left(t_{1}\right), z_{1 i}\left(t_{1}\right)\right]^{T} \notin Q_{i}$ in number $j_{i}$ subspace, where $t_{1}>0$. Since point $\mathbf{z}(0) \in Q_{i}=\left\{\left(z_{2 i}, z_{1 i}\right) \mid H_{k i}(z) \geq 0, k_{i}=0,1,2,3\right\}$ in number $k_{i}$ subspace and $M_{i}>0$, it is noted that the Lyapunov function $V_{i}(\cdot)$ in (A.1) can be expressed as follows:

$$
V_{i}(z(0))=\frac{1}{2}\left[\frac{\left(M_{i}-H_{k i}(z(0))\right)}{M_{i}}\right]^{2} \leq \frac{1}{2} .
$$

Since point $\mathbf{z}\left(t_{1}\right)=\left[z_{2 i}\left(t_{1}\right), z_{1 i}\left(t_{1}\right)\right]^{T} \notin Q_{i}$ in number $j_{i}$ subspace, there exists $H_{j i}\left(\mathbf{z}\left(t_{1}\right)\right)<0$ from Remark 6. Clearly, the conclusion in (A.2) is obtained as

$$
\begin{aligned}
H_{j i}\left(\mathbf{z}\left(t_{1}\right)\right) & <0, \\
M_{i} & >0, \\
V_{i}\left(z\left(t_{1}\right)\right) & =\frac{1}{2}\left[\frac{\left(M_{i}-H_{j i}\left(z\left(t_{1}\right)\right)\right)}{M_{i}}\right]^{2} \leq \frac{1}{2} .
\end{aligned}
$$

It follows from (A.11) and (A.12) that the continuous Lyapunov function $V_{i}(\cdot)$ has the result $V_{i}(\mathbf{z}(0))<V_{i}\left(\mathbf{z}\left(t_{1}\right)\right), 0<t_{1}$. Hence, there should exist a point $\mathbf{z}\left(t_{2}\right), 0<t_{2}<t_{1}$, where $\dot{V}_{i}\left(\mathbf{z}\left(t_{2}\right)\right)>0$. However, from the above discussion in Part (1), it is noted that $\dot{V}_{i}<0$. Then, we find the contradiction since the derivative of Lyapunov function $V_{i}(\cdot)$ cannot be positive and negative at the same time on the point $\mathbf{z}\left(t_{2}\right)$. Hence, the assumption that there exists $\mathbf{z}\left(t_{1}\right) \notin Q_{i}$ is incorrect, and $\mathbf{z}(0) \in Q_{i}$ implies $\mathbf{z}(t) \in Q_{i}$ for all $t>0$. From Definition 5 , the set $Q_{i}=\left\{\left(z_{2 i}, z_{1 i}\right) \mid H_{k i}(\mathbf{z}) \geq 0, k_{i}=0,1,2,3\right\}$ is a positively invariant set. This completes the proof.

\section{Conflict of Interests}

The authors declare that there is no conflict of interests regarding the publication of this paper.

\section{Acknowledgments}

The authors wish to thank the editor and anonymous reviewers for useful comments and suggestions. This work is partially supported by JSUT Research Funding (Grant nos. KYY13001, KYY13017) and Innovation Team Funding (Grant no. TDZD13003).

\section{References}

[1] M. Young, S. Keith, and A. Pancotti, "An overview of advanced concepts for near-space systems," in Proceedings of the 45th AIAA/ASME/SAE/ASEE Joint Propulsion Conference and Exhibit, pp. 1-8, August 2009.

[2] M. Chen and B. Jiang, "Robust attitude control of near space vehicles with time-varying disturbances," International Journal of Control, Automation and Systems, vol. 11, no. 1, pp. 182-187, 2013.

[3] J. Zhao, B. Jiang, P. Shi, Z. Gao, and D. Xu, "Fault-tolerant control design for near-space vehicles based on a dynamic terminal sliding mode technique," Proceedings of the Institution of Mechanical Engineers. Part I: Journal of Systems and Control Engineering, vol. 226, no. 6, pp. 787-794, 2012.

[4] J. Zhao, B. Jiang, P. Shi, and Z. He, "Fault tolerant control for damaged aircraft based on sliding mode control scheme," International Journal of Innovative Computing, Information and Control, vol. 10, no. 1, pp. 293-302, 2014.

[5] J. He, R. Qi, B. Jiang, and J. Qian, "Adaptive output feedback fault-tolerant control design for hypersonic flight vehicles," Journal of the Franklin Institute. Engineering and Applied Mathematics, vol. 352, no. 5, pp. 1811-1835, 2015.

[6] R. Zhang, L. Dong, and C. Sun, "Adaptive nonsingular terminal sliding mode control design for near space hypersonic vehicles," IEEE/CAA Journal of Automatica Sinica, vol. 1, no. 2, pp. 155-161, 2014.

[7] M. Pu, Q. X. Wu, C. S. Jiang, and J. Zhang, "Near space vehicle control based on second-order dynamic terminal sliding mode," The Journal of Astronautics, vol. 31, no. 4, pp. 1056-1062, 2010.

[8] Y. L. Du, Q. X. Wu, C. S. Jiang, and J. Wen, "Adaptive functional link network control of near-space vehicles with dynamical uncertainties," Journal of Systems Engineering and Electronics, vol. 21, no. 5, pp. 868-876, 2010.

[9] D. X. Gao and Z. Q. Sun, "Fuzzy tracking control design for hypersonic vehicles via T-S model," Science China Information Sciences, vol. 54, no. 3, pp. 521-528, 2011.

[10] B. Jiang, Z. Gao, P. Shi, and Y. Xu, "Adaptive fault-tolerant tracking control of near-space vehicle using Takagi-Sugeno fuzzy models," IEEE Transactions on Fuzzy Systems, vol. 18, no. 5, pp. 1000-1007, 2010.

[11] F. Wang, C. Hua, and Q. Zong, "Attitude control of reusable launch vehicle in reentry phase with input constraint via robust adaptive backstepping control," International Journal of Adaptive Control and Signal Processing, 2015.

[12] Q. Zong, F. Wang, B. Tian, and R. Su, "Robust adaptive approximate backstepping control of a flexible air-breathing hypersonic vehicle with input constraint and uncertainty," Proceedings of the Institution of Mechanical Engineers. Part I: Journal of Systems and Control Engineering, vol. 228, no. 7, pp. 521-539, 2014.

[13] M. Chen and B. Jiang, "Robust bounded control for uncertain flight dynamics using disturbance observer," Journal of Systems Engineering and Electronics, vol. 25, no. 4, Article ID 6905958, pp. 640-647, 2014. 
[14] X. Wei and L. Guo, "Composite disturbance-observer-based control and $H_{\infty}$ control for complex continuous models," International Journal of Robust and Nonlinear Control, vol. 20, no. 1, pp. 106-118, 2010.

[15] W. H. Chen, "Nonlinear disturbance observer-enhanced dynamic inversion control of missiles," Journal of Guidance, Control, and Dynamics, vol. 26, no. 1, pp. 161-166, 2003.

[16] F. Chen, F. Lu, B. Jiang, and G. Tao, "Adaptive compensation control of the quadrotor helicopter using quantum information technology and disturbance observer," Journal of the Franklin Institute, vol. 351, no. 1, pp. 442-455, 2014.

[17] L. Wu, P. Shi, and X. Su, Sliding Mode Control of Uncertain Parameter-Switching Hybrid Systems, John Wiley \& Sons, Chichester, UK, 2014.

[18] Y. Wang, Q. Zhang, and L. Zhu, "Active control of hypersonic airfoil flutter via adaptive fuzzy sliding mode method," Journal of Vibration and Control, vol. 21, no. 1, pp. 134-141, 2015.

[19] L. Yan and B. S. Ju, "PMSM speed controller using switching algorithm of $\mathrm{PD}$ and sliding mode control," in Proceedings of the ICROS-SICE International Joint Conference, pp. 12601266, Fukuoka International Congress Center, Fukuoka, Japan, August 2009.

[20] M. S. Chen, Y. R. Hwang, and M. Tomizuka, "A state-dependent boundary layer design for sliding mode control," IEEE Transactions on Automatic Control, vol. 47, no. 10, pp. 1677-1681, 2002.

[21] V. S. Bandal and P. N. Vernekar, "Design of a discrete-time sliding mode controller for a magnetic levitation system using multirate output feedback," in Proceedings of the American Control Conference (ACC '10), pp. 4289-4294, Baltimore, Md, USA, July 2010.

[22] R. Zhang, C. Sun, J. Zhang, and Y. Zhou, "Second-order terminal sliding mode control for hypersonic vehicle in cruising flight with sliding mode disturbance observer," Journal of Control Theory and Applications, vol. 11, no. 2, pp. 299-305, 2013.

[23] J. Yang, S. Li, C. Sun, and L. Guo, "Nonlinear-disturbanceobserver-based robust flight control for airbreathing hypersonic vehicles," IEEE Transactions on Aerospace and Electronic Systems, vol. 49, no. 2, pp. 1263-1275, 2013.

[24] J. Fu, Q. X. Wu, C. S. Jiang, and Y. Wang, "Robust sliding mode positively invariant set control for a class of nonlinear continuous systems," Acta Automatica Sinica, vol. 37, no. 11, pp. 1395-1401, 2011.

[25] J. Fu, Q. X. Wu, C. S. Jiang, and L. Cheng, "Robust sliding mode control with unidirectional auxiliary surfaces for a nonlinear system with state constraints," Control and Decision, vol. 26, no. 9, pp. 1288-1294, 2011.

[26] W. I. Ai, Y. H. Wang, L. Zhu, and C. Xu, "Design of sliding mode controller with unidirectional auxiliary surfaces based on improved exponent trending law for post-stall maneuver," Journal of Jilin University (Information Science Edition), vol. 33, no. 2, pp. 153-160, 2015.

[27] T. W. Hu, Q. X. Wu, C. S. Jiang et al., "Exponential approach law for sliding mode control with unidirectional auxiliary surfaces," Electronics Optics \& Control, vol. 20, no. 7, pp. 36-41, 2013.

[28] S. A. Snell, Nonlinear dynamic-inversion flight control of supermaneuverable aircraft [Ph.D. thesis], University of Minnesota, 1991.

[29] M. Chen, B. Jiang, Q. X. Wu, and C. Jiang, "Robust control of near-space vehicles with input backlash-like hysteresis," Proceedings of the Institution of Mechanical Engineers, Part I: Journal of Systems and Control Engineering, vol. 227, no. 8, pp. 635-644, 2013.
[30] F. Blanchini, "Set invariance in control," Automatica, vol. 35, no. 11, pp. 1747-1767, 1999.

[31] Y. Feng, X. Yu, and F. Han, "On nonsingular terminal slidingmode control of nonlinear systems," Automatica, vol. 49, no. 6, pp. 1715-1722, 2013.

[32] W. H. Chen, "Disturbance observer based control for nonlinear systems," IEEE/ASME Transactions on Mechatronics, vol. 9, no. 4, pp. 706-710, 2004. 


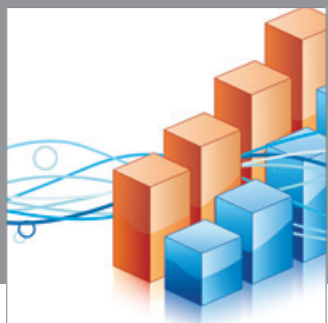

Advances in

Operations Research

mansans

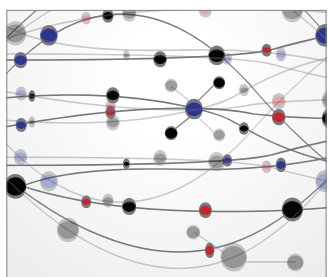

The Scientific World Journal
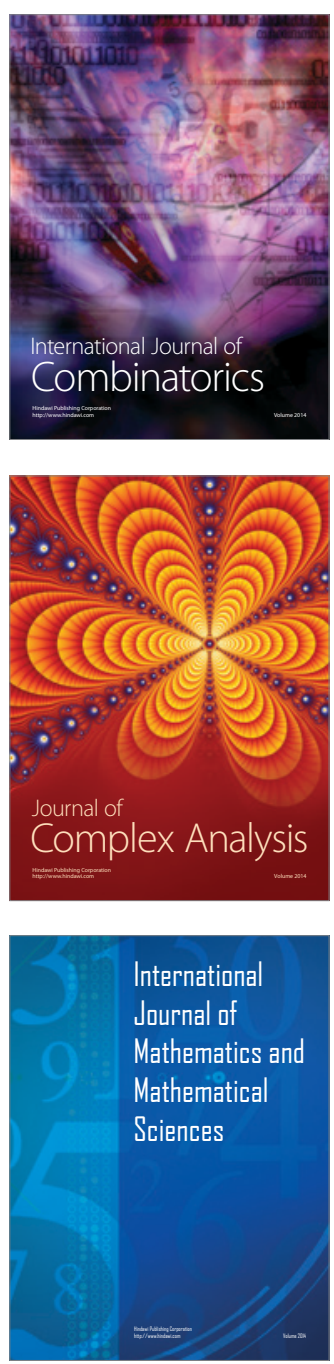
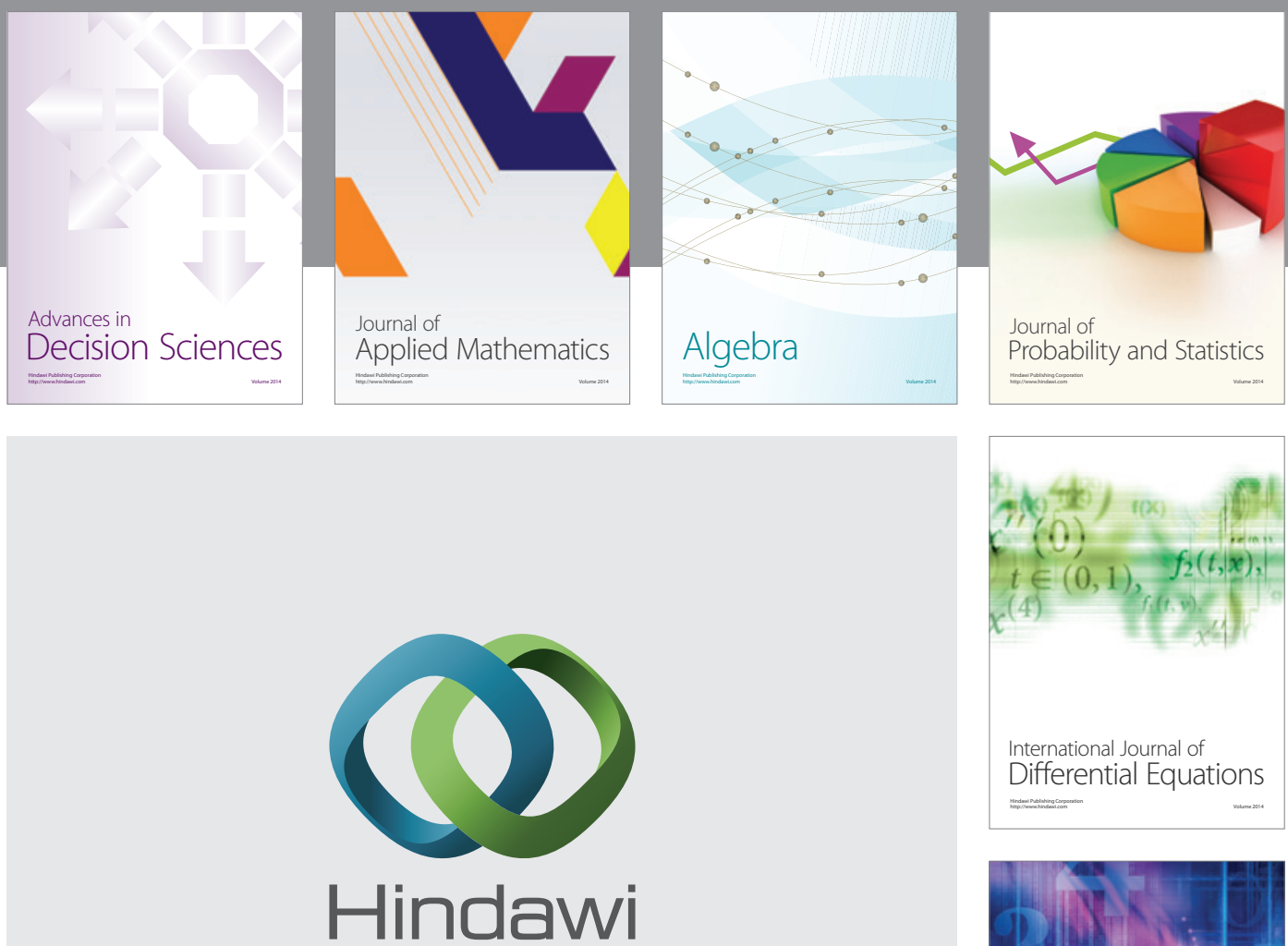

Submit your manuscripts at http://www.hindawi.com
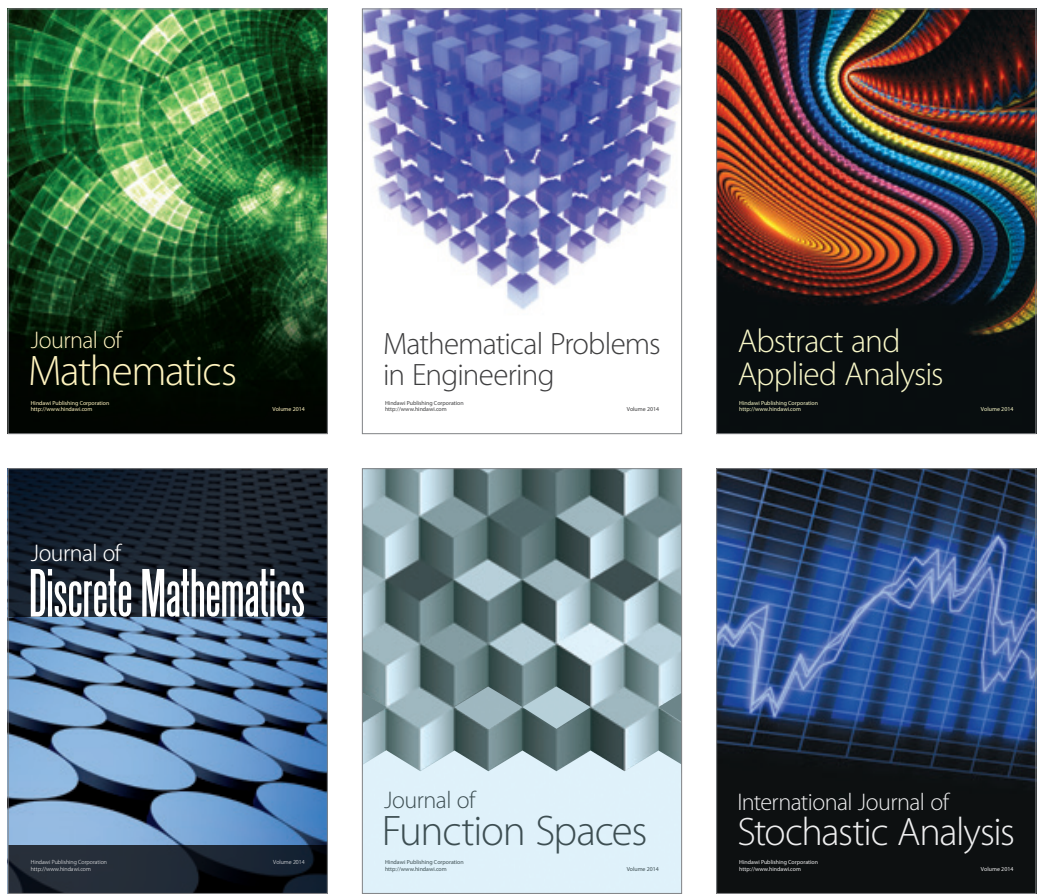

Journal of

Function Spaces

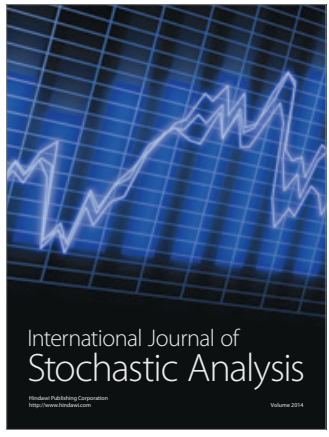

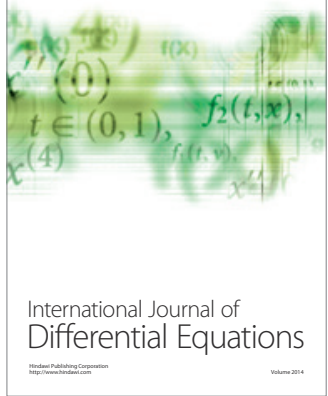
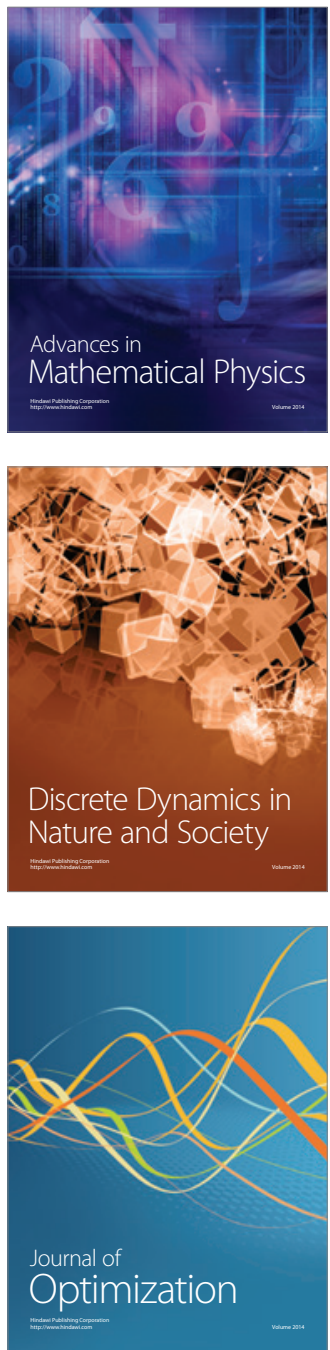